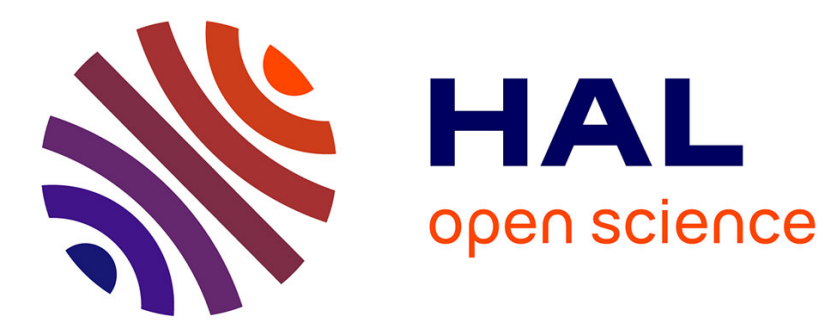

\title{
Extension de l'analyse simultanée des bandes v2 et v 4 de $12 \mathrm{CH} 4$
}

\author{
Charles Pierre, G. Pierre, Jean-Paul Champion, B.L. Lutz
}

\section{To cite this version:}

Charles Pierre, G. Pierre, Jean-Paul Champion, B.L. Lutz. Extension de l'analyse simultanée des bandes v2 et v 4 de 12CH4. Journal de Physique Lettres, 1980, 41 (14), pp.319-322. 10.1051/jphyslet:019800041014031900 . jpa-00231789

\section{HAL Id: jpa-00231789 https://hal.science/jpa-00231789}

Submitted on 1 Jan 1980

HAL is a multi-disciplinary open access archive for the deposit and dissemination of scientific research documents, whether they are published or not. The documents may come from teaching and research institutions in France or abroad, or from public or private research centers.
L'archive ouverte pluridisciplinaire HAL, est destinée au dépôt et à la diffusion de documents scientifiques de niveau recherche, publiés ou non, émanant des établissements d'enseignement et de recherche français ou étrangers, des laboratoires publics ou privés. 


\title{
LE JOURNAL DE PHYSIQUE-LETTRES
}

\section{Extension de l'analyse simultanée des bandes $\nu_{2}$ et $\nu_{4}$ de ${ }^{12} \mathrm{CH}_{4}$}

\author{
C. Pierre, G. Pierre, J. P. Champion \\ Laboratoire de Spectronomie Moléculaire (*), Faculté des Sciences, 6, bd Gabriel, 21100 Dijon, France
}

et B. L. Lutz

Planetary Research Center, Lowell Observatory, Box 1269, Flagstaff, Arizona 86002, U.S.A.

(Reçu le 6 mai 1980, accepté le 27 mai 1980)

\begin{abstract}
Résumé. - Une nouvelle analyse simultanée des bandes $v_{2}$ et $v_{4}$ de ${ }^{12} \mathrm{CH}_{4}$ a été réalisée à partir de spectres IR d'une résolution de $0,05 \mathrm{~cm}^{-1} .973$ transitions sont attribuées aux deux bandes de P20 à $\mathrm{R} 18$. Elles sont reproduites avec un écart quadratique moyen de $17 \mathrm{mK}$ à l'aide d'un hamiltonien limité à l'ordre 3 pour les termes diagonaux comme pour les termes d'interaction. Ce modèle ne comporte que 16 paramètres à ajuster qui sont tous déterminés avec une précision relative de l'ordre du centième.
\end{abstract}

\begin{abstract}
A new simultaneous analysis of the $v_{2}$ and $v_{4}$ bands of ${ }^{12} \mathrm{CH}_{4}$ has been carried out from infrared spectra recorded with a resolution of $0.05 \mathrm{~cm}^{-1} .973$ transitions have been assigned to these two bands from P20 to R18. They have been fitted using a third order hamiltonian for diagonal terms as well as for off-diagonal terms. This model involves only 16 adjustable parameters which are all determined with an accuracy of the order of one per cent. The standard deviation of the fit is $17 \mathrm{mK}$.
\end{abstract}

1. Introduction. - Les diverses analyses simultanées des bandes $v_{2}$ et $v_{4}$ de ${ }^{12} \mathrm{CH}_{4}$ déjà publiées $[1,2$, 3] sont essentiellement limitées par la précision insuffisante des données expérimentales sur la bande $v_{2}$. En effet, jusqu'à présent, les seules données utilisées pour cette bande provenaient du spectre Raman enregistré à Dijon avec une résolution de $0,10 \mathrm{~cm}^{-1}[4]$.

Dans cet article nous présentons une étude de ces bandes plus complète et plus précise grâce à l'exploitation de spectres infrarouges du méthane dans la région de 1120 à $1800 \mathrm{~cm}^{-1}$ enregistrés sur un spectromètre à transformée de Fourier [5] avec une résolution de $0,05 \mathrm{~cm}^{-1}$.

Plus de 900 transitions des bandes $v_{2}$ et $v_{4}$, correspondant à 800 raies expérimentales, sont reproduites avec un écart quadratique moyen de $17 \mathrm{mK}$. A titre de comparaison, les études antérieures les plus récentes [2,3] ne traitent qu'environ 500 raies avec un écart quadratique moyen sensiblement supérieur. Ce travail constitue la première phase du dépouillement complet de cette région spectrale $\mathrm{du}$

(*) Equipe de recherche associée au C.N.R.S. méthane qui comporte notamment un grand nombre de transitions provenant de bandes chaudes [6]. L'intérêt de cette étude dans le domaine de l'astrophysique en particulier a déjà été évoqué par ailleurs [5, 7].

La bonne précision des données expérimentales incluant des transitions relatives à des valeurs du nombre quantique $J$ relativement élevées $(J \leqslant 19)$ pour les deux bandes, nous a permis d'améliorer sensiblement le modèle théorique utilisé. Nous avons développé l'hamiltonien rigoureusement au troisième ordre dans la classification de Amat et Nielsen [8] pour les termes d'interaction comme pour les termes diagonaux en $v$. Cela implique seulement 16 paramètres à déterminer au lieu de 24 ou 21 dans les études récentes réalisées par Gray et Robiette [2] et Champion [3] respectivement. Contrairement à ces dernières analyses dans lesquelles le coefficient de Coriolis $\zeta_{24}$ était fixé a priori, les 16 paramètres utilisés ici sont tous déterminés directement à partir des fréquences observées avec une précision relative de l'ordre du centième.

Dans cet article, nous nous limiterons à un rappel sommaire de la théorie pour donner une plus grande place à la discussion des résultats. 
2. Données expérimentales. - Les données expérimentales utilisées dans ce travail proviennent de trois spectres infrarouges couvrant la région de 1120 à $1800 \mathrm{~cm}^{-1}$ avec une résolution de $0,05 \mathrm{~cm}^{-1}$ et des abondances de $0,765,3,78$ et $15,5 \mathrm{~cm}$-atm respectivement [5]. La précision sur la position des raies est estimée à $\pm 0,005 \mathrm{~cm}^{-1}$. Pour une description plus complète, le lecteur intéressé pourra se reporter à la référence [5]. Dans le cadre de la présente étude, ayant pour objectif le dépouillement complet de la région spectrale, l'élimination des raies d'absorption dues à la vapeur d'eau résiduelle a été améliorée en rapportant chacun des spectres du méthane au spectre approprié d'une cellule vide.

Nous avons utilisé ces données à l'exclusion en particulier des mesures plus précises publiées récemment par Restelli et Cappellani [9] et Pinson et DupréMaquaire [10] sur la branche $Q$ de $v_{4}$. En effet, pour les raisons exposées dans les paragraphes suivants, nous avons retenu un modèle théorique qui donne priorité aux termes d'interaction. En conséquence, la prise en compte de données plus précises sur une branche particulière d'une seule des deux bandes en interaction a un effet négligeable sur la précision des paramètres. Pour la même raison nous n'avons pas repris les données expérimentales partielles utilisées à la référence [6].

3. Modèle théorique. - La théorie utilisée, basée sur le formalisme tensoriel dans le groupe de symétrie de la molécule (groupe $T_{d}$ ), ainsi que la méthode d'analyse proprement dite, ont été déjà largement exposées aux références [3] et [11]. Nous nous bornerons ici à montrer la modification apportée au modèle introduit par Champion [3].

Les constantes du niveau de base du méthane sont connues avec une grande précision [12]. Au quatrième ordre d'approximation, ces constantes sont au nombre de 6 : elles correspondent à la deuxième colonne du tableau I. Les termes rovibrationnels sont classés en 3 catégories : les termes relatifs à la vibration $v_{4}$, les termes relatifs à la vibration $v_{2}$ et les termes d'interaction entre ces deux modes de vibration. Au troisième ordre, ces trois catégories comportent respectivement 7, 4 et 5 termes (colonnes 3,4 et 5 du tableau I). Les termes d'ordre quatre sont au nombre de 6,4 et 3 respectivement.

L'analyse réalisée par Champion [3] fait intervenir tous les opérateurs situés au-dessus de la ligne en pointillés du tableau I. En particulier, l'hamiltonien d'interaction est limité à l'ordre deux. Ce modèle était justifié par le fait que les données expérimentales n'atteignaient pas des valeurs de $J$ très élevées (notamment pour la bande $v_{2}, J<11$ ) et il était illusoire de chercher à déterminer des termes d'interaction d'ordre trois de la forme $P^{3} q_{2} q_{4}$. En effet pour des valeurs de $J$ voisines de 10 , un calcul de perturbation au premier ordre montre que la contribution énergétique de ces termes est inférieure au centième de $\mathrm{cm}^{-1}$.
Tableau I. - Nomenclature et ordre de grandeur des termes de H. $\Omega=$ degré par rapport aux composantes $P_{\alpha} d u$ moment angulaire. $K=$ rang tensoriel dans $\mathrm{SO}(3) . \quad \Gamma=$ représentation irréductible de $\mathrm{T}_{\mathrm{d}} . L e$ tableau donne l'ordre de grandeur de chaque terme suivant la classification de Amat et Nielsen.

[Nomenclature and order of magnitude of the Hamiltonian terms. $\Omega=$ degree in angular momentum components $P_{\alpha} . K=$ tensor rank in $\mathrm{SO}(3) . \Gamma=$ irreducible representation of $T_{d}$. The table gives the order of magnitude of each term according Amat and Nielsen classification.]

\begin{tabular}{|c|c|c|c|c|}
\hline $\begin{array}{c}v^{(\Gamma)} \\
\Omega(K, \Gamma)\end{array}$ & $I^{(A 1)}$ & $V_{4,4}^{F 2 ~ F 2(\Gamma)}$ & $\begin{array}{l}E E(\Gamma) \\
V_{2,2}\end{array}$ & $\begin{array}{l}E F 2(\Gamma) \\
V_{2,4}\end{array}$ \\
\hline $0(0, A 1)$ & $\bullet$ & 0 & 0 & \\
\hline $1(1, F 1)$ & & 1 & & 1 \\
\hline $2(0, A 1)$ & 0 & 2 & 2 & \\
\hline $2(2, E)$ & & 2 & 2 & \\
\hline $2(2, F 2)$ & & 2 & & 2 \\
\hline $3(1, F 1)$ & & 3 & & 1 \\
\hline $3(3, A 2)$ & & & 3 & 1 \\
\hline $3(3, F 1)$ & & 3 & & i \\
\hline $3(3, F 2)$ & & & & 3 \\
\hline $4(0, A 1)$ & 2 & 4 & 4 & 1 \\
\hline $4(2, E)$ & & 4 & 4 & $\begin{array}{l}1 \\
1\end{array}$ \\
\hline $4(2, F 2)$ & & 4 & & $i$ \\
\hline $4(4, A 1)$ & 2 & 4 & 4 & 1 \\
\hline $4(4, E)$ & & 4 & 4 & i \\
\hline $4(4, F 1)$ & & & & 1 \\
\hline $4(4, F 2)$ & & 4 & & 4 \\
\hline $6(0, A 1)$ & 4 & & & \\
\hline $6(4, A 1)$ & 4 & & & \\
\hline $6(6, A 1)$ & 4 & & & \\
\hline Type & $p^{\Omega}$ & $\mathrm{p}^{\Omega} \mathrm{q}_{4}^{2}$ & $p^{\Omega} q_{2}^{2}$ & $\mathrm{P}^{\Omega} \mathrm{q}_{2} \mathrm{q}_{4}$ \\
\hline
\end{tabular}

Le modèle utilisé par Gray et Robiette [2] est très semblable, à cette différence près que ces auteurs ne considèrent qu'un seul opérateur d'interaction, le terme de Coriolis, et introduisent quelques termes diagonaux d'ordre supérieur à quatre.

Dans les deux cas, les valeurs effectives des paramètres diagonaux d'ordre quatre ou plus prennent implicitement en compte les termes d'interaction négligés.

Toutefois, lorsqu'on veut rendre compte de transitions atteignant des valeurs de $J$ de l'ordre de 19 . les termes d'interaction ont une contribution analogue à celle des termes diagonaux du même ordre. Cela est particulièrement bien illustré sur le diagramme des niveaux d'énergie (Fig. 1) où l'on constate que pour $J=19$, la distance minimale entre les niveaux $v_{2}=1$ et $v_{4}=1$ est du même ordre de grandeur que l'éclatement du niveau $v_{4}=1$ dû principalement au terme de Coriolis $B \zeta_{4}$. La même situation se 


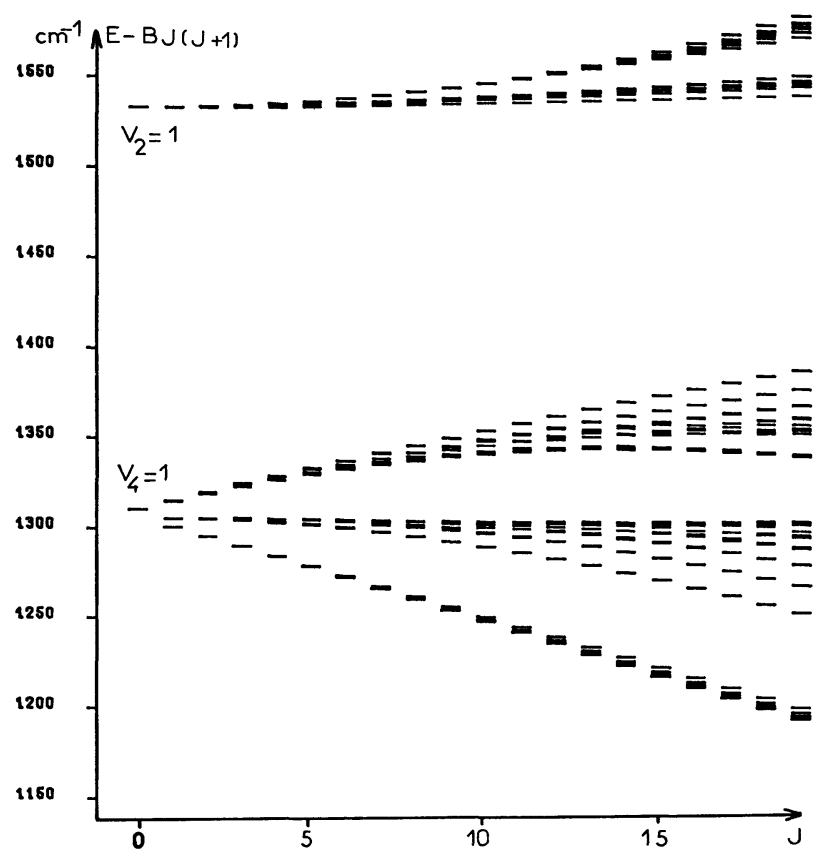

Fig. 1. - Diagramme des niveaux d'énergie de $v_{2}$ et $v_{4}$ de ${ }^{12} \mathrm{CH}_{4}$. [Diagram of energy levels of $v_{2}$ and $v_{4}$ of ${ }^{12} \mathrm{CH}_{4}$.]

retrouve, plus accentuée encore, dans la molécule de ${ }^{28} \mathrm{SiH}_{4}[13,14]$ par exemple. Dans leur étude simultanée de $v_{2}$ et $v_{4}$ de ${ }^{28} \mathrm{SiH}_{4}$, Gray et Robiette [14] éprouvent d'ailleurs la nécessité d'ajouter à leur modèle un terme d'interaction d'ordre trois obtenu en multipliant le terme de Coriolis par $P^{2}$. Le choix de ce terme particulier, à l'exclusion du terme d'ordre deux (voir Réf. [13]) ainsi que des deux autres termes d'ordre 3 , résulte davantage de considérations pratiques liées à la complexité relative du calcul de ces divers termes plutôt que de considérations purement physiques.

En ce qui concerne le méthane, dans ce travail, nous avons considéré tous les termes d'interaction jusqu'à l'ordre trois. Par rapport au modèle précédent [3] les trois nouveaux termes sont les suivants : $T_{2,4}^{3(1, F 1) E ~ F 2}$, $T_{2,4}^{3(3, F 1) \mathrm{EF} 2}$, et $T_{2,4}^{3(3, \mathrm{~F} 2) \mathrm{EF} 2}$. La forme explicite de ces opérateurs, ainsi que le calcul de leurs éléments matriciels, sont décrits aux références [3] et [11]. A ce propos, il convient de remarquer la grande souplesse $\mathrm{du}$ formalisme tétraédrique qui donne directement par une formule unique (équation (30) de la référence [3]) les éléments matriciels de tous les opérateurs possibles, c'est-à-dire totalement symétriques par rapport à l'espace et au temps.

4. Résultats. - Utilisant les données expérimentales décrites au paragraphe 2 , nous avons commencé la nouvelle analyse en ajoutant simplement au modèle précédent les 3 nouveaux termes d'interaction mentionnés plus haut. Le terme de Coriolis

$$
\tilde{t}_{2,4}^{1(1, \mathrm{~F} 1) \mathrm{EF} 2}=-3 B \zeta_{24}
$$

restant fixé, après quelques cycles d'ajustement par une méthode classique de moindres carrés non pondérée, nous avons obtenu un écart quadratique moyen entre transitions observées et calculées sensiblement amélioré. En contrepartie, des corrélations importantes apparurent notamment entre ces nouveaux opérateurs et les termes diagonaux d'ordre quatre. Nous avons alors essayé de limiter le développement de l'hamiltonien strictement à l'ordre trois pour les termes diagonaux comme pour les termes d'interaction. Ce modèle englobe les termes situés au-dessus de la ligne horizontale continue du tableau I. Nous avons cependant conservé les 6 constantes du niveau de base à l'ordre quatre puisque ces dernières ont fait l'objet d'une détermination simultanée et qu'elles restent fixées au cours de l'ajustement des autres paramètres. Cette réduction importante du nombre global de paramètres n'a entraîné qu'une remontée relativement faible de l'écart quadratique moyen $\left(0,017 \mathrm{~cm}^{-1}\right)$, largement compensée par la disparition de toutes les corrélations importantes. Il a donc été possible de libérer tous les paramètres, y compris ceux d'interaction, sans qu'il soit nécessaire de recourir à un quelconque artifice pour assurer la convergence de la méthode de moindres carrés.

Tous les paramètres ont été déterminés avec une déviation standard inférieure au centième de leur valeur absolue à l'exception du terme $\widetilde{t}_{2,4}^{3(1, F 1) E F 2}$ dont la précision est de l'ordre de $3 \%$ (Tableau II).

L'écart quadratique moyen sur les transitions peut être amélioré en réintroduisant des termes diagonaux en $v$ d'ordre quatre, à condition d'assurer la convergence du processus d'ajustement par exemple en fixant à nouveau le terme prépondérant d'interaction $\left({ }^{1}\right)$.

Toutefois, la limitation stricte à l'ordre trois nous paraît fournir le modèle le mieux adapté en vue de l'exploitation des résultats pour d'autres études en cours de réalisation. En effet, la raison principale de notre choix résulte de considérations liées à l'analyse des harmoniques et des combinaisons des modes $v_{2}$ et $v_{4}$. L'interaction entre les bandes $v_{2}$ et $v_{4}$ intervient au niveau des bandes $2 v_{2}, v_{2}+v_{4}$ et $2 v_{4}$ de manière encore plus importante. Ce qui explique par exemple que les analyses séparées de ces dernières n'ont pu être réalisées que pour des valeurs de $J$ inférieures ou égales à 7 [15]. Aussi est-il logique de penser que l'extrapolation des résultats de $v_{2}$ et $v_{4}$ pour l'analyse des bandes $2 v_{2}, v_{2}+v_{4}$ et $2 v_{4}$ sera plus efficace en utilisant un modèle qui donne priorité aux termes d'interaction plutôt qu'un modèle dans lequel, du fait des corrélations importantes, une partie de l'interaction est prise en compte implicitement par les valeurs

( $\left.{ }^{1}\right)$ Ce travail étant achevé, nous avons eu connaissance d'une étude en cours de réalisation par A. G. Robiette. Ce dernier, reprenant pratiquement le même modèle que celui des références [2] et [3] et introduisant des données expérimentales plus variées, obtient par une méthode de moindres carrés pondérée un écart quadratique moyen plus petit. 
Tableau II. - Coefficients de l'hamiltonien relatifs $\grave{a} v_{2}$ et $v_{4}$ de ${ }^{12} \mathrm{CH}_{4}$. Les valeurs sont exprimées en $\mathrm{cm}^{-1}$. $L$ 'incertitude standard sur les deux derniers chiffres est indiquée entre parenthèses.

[Hamiltonian coefficients for $v_{2}$ and $v_{4}$ of ${ }^{12} \mathrm{CH}_{4}$. The values are in $\mathrm{cm}^{-1}$. The standard error on the two last figures is indicated in parenthesis.]

\begin{tabular}{|c|c|c|c|c|}
\hline$\Omega(K, \Gamma)$ & $t_{4,4}^{\Omega(K, r) F 2}$ & $\mathrm{~F} 2$ & $\mathrm{t}_{2,2}^{\Omega(K, \Gamma) E \mathrm{E}}$ & $\mathrm{t}_{2,4}^{\Omega(K, r) E \quad F 2}$ \\
\hline $0(0, A 1)$ & 1310,7556 & (17) & $1533.3455(21)$ & \\
\hline $1(1, F 1)$ & 10,34721 & $1(38)$ & & $-9,6334(18)$ \\
\hline $2(0, A 1)$ & $-0,1813$ & (23) $\times 10^{-2}$ & $-0,6640(35) \times 10^{-2}$ & \\
\hline $2(2, E)$ & $-0,9418$ & (34) $\times 10^{-2}$ & $-3,1455(29) \times 10^{-2}$ & \\
\hline $2(2, F 2)$ & $-2,7579$ & $(13) \times 10^{-2}$ & & $-3,570(15) \times 10^{-2}$ \\
\hline $3(1, F 1)$ & 0,5245 & $(23) \times 10^{-3}$ & & $-0,0828(25) \times 10^{-3}$ \\
\hline $3(3, A 2)$ & & & $-0,2099(18) \times 10^{-3}$ & \\
\hline $3(3, F 1)$ & 0,2362 & $(22) \times 10^{-3}$ & & $0,2300(15) \times 10^{-3}$ \\
\hline $3(3, F 2)$ & & & & $0,1983(26) \times 10^{-3}$ \\
\hline
\end{tabular}

efficaces des termes diagonaux d'ordre élevé. Ajoutons que l'analyse des bandes $2 v_{2}, v_{2}+v_{4}$ et $2 v_{4}$ impose de traiter en même temps les bandes $v_{1}$ et $v_{3}$, ce qui représente une dégénérescence vibrationnelle totale de 19. Pour ce type d'étude il est particulièrement important d'utiliser le modèle qui introduit le plus petit nombre possible de paramètres.

Enfin, aucun paramètre n'étant fixé à une valeur déterminée par ailleurs, la présente analyse fournit une détermination expérimentale directe des coefficients de l'hamiltonien d'interaction entre $v_{2}$ et $v_{4}$. Compte tenu de l'état actuel des connaissances sur le champ de force de la molécule, essentiellement limitées aux termes cubiques du potentiel [16], il est plus utile de connaître les valeurs expérimentales de paramètres d'ordre peu élevé plutôt que de termes diagonaux d'ordre plus élevé dont la signification physique est beaucoup plus délicate à établir. Remarquons que la valeur expérimentale obtenue pour le coefficient de
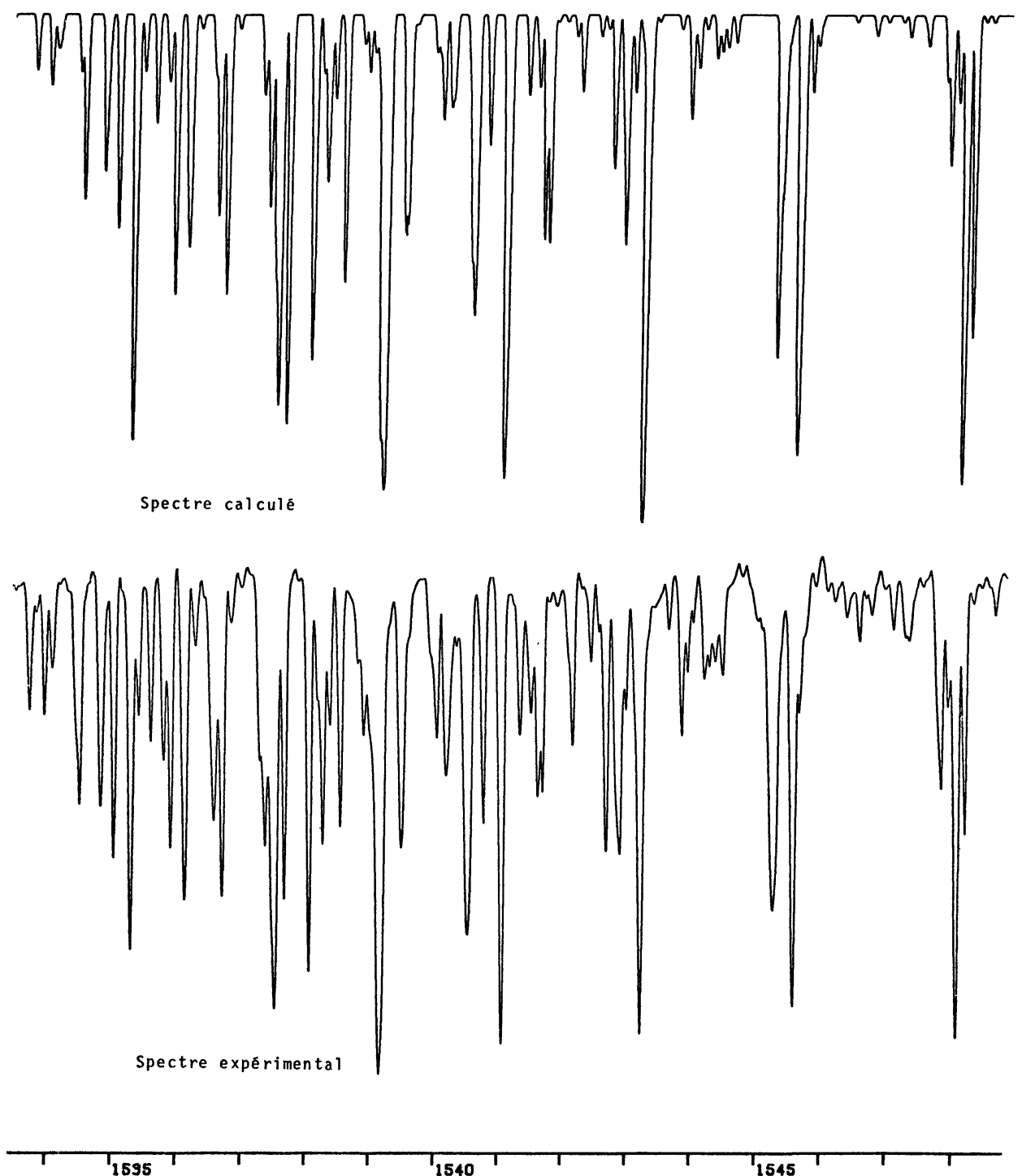

Fig. 2. - Branche $\mathrm{Q}$ de la bande $v_{2} \mathrm{de}^{12} \mathrm{CH}_{4}$.

[Q branch of the $v_{2}$ band of ${ }^{12} \mathrm{CH}_{4}$.] 
Coriolis $B \zeta_{24}=(3,2111 \pm 0,0006) \mathrm{cm}^{-1}$ est un peu supérieure à la valeur $3,162 \mathrm{~cm}^{-1}$ calculée par Gray et Robiette [16] à partir du champ de force. On pourra comparer les valeurs des autres paramètres avec celles des études précédentes en utilisant le tableau IV de la référence [11].

Nous avons attribué au total 973 transitions de P20 à $\mathrm{R} 18$. Elles sont reproduites avec un écart quadratique moyen de $0,017 \mathrm{~cm}^{-1}$. Pour des raisons d'ordre pratique, la liste de ces transitions sera publiée ultérieurement en même temps que les attributions des bandes chaudes $2 v_{4}-v_{4}$ et $v_{2}+v_{4}-v_{2}$ en cours d'étude. Elle peut cependant être obtenue dès maintenant en écrivant aux auteurs.

La figure 2 illustre l'accord satisfaisant entre spectres expérimental et calculé dans une région particulièrement dense, la branche $Q$ de la bande $v_{2}$. Celle-ci étant interdite en première approximation, les intensités sont calculées exclusivement à partir $\mathrm{du}$ moment dipolaire associé à la vibration $v_{4}$ et résultent de l'interaction entre les deux bandes. La plupart des dissemblances apparaissent pour des raies composées de plusieurs transitions. Elles sont généralement imputables au fait que le profil de Gauss utilisé pour dessiner le spectre calculé est une approximation assez grossière du profil expérimental.
5. Conclusion. - Les résultats présentés dans cet article ont un intérêt à la fois pratique et théorique. Sur le plan pratique, ils constituent la première étape d'une analyse plus complète de cette région spectrale du méthane. L'attribution précise de toutes les raies faibles ou intenses appartenant aux bandes $v_{2}$ et $v_{4}$ était indispensable avant l'étude des bandes chaudes. Sur le plan théorique, notre travail met en évidence les avantages du traitement rigoureux des interactions entre bandes. Le même modèle s'applique avec la même efficacité aux bandes $v_{1}$ et $v_{3}$ d'une part, $v_{2}$ et $v_{4}$ d'autre part de la molécule ${ }^{28} \mathrm{SiH}_{4}$ [17]. Cette molécule est l'exemple type pour lequel la prise en compte de tous les termes d'interaction n'est pas seulement avantageux mais réellement indispensable. Comme pour le méthane, la limitation du développement de l'hamiltonien à l'ordre 3 est possible, minimisant ainsi le nombre de paramètres nécessaires.

Remerciements. - Les auteurs remercient A. G. Robiette pour la communication de ses résultats avant publication. B. L. Lutz remercie «the National Aeronautics and Space Administration » pour l'aide apportée (grant NSG-7499).

\section{Bibliographie}

[1] Berger, H., J. Mol. Spectrosc. 55 (1975) 48-55.

[2] Gray, D. L. et Robiette, A. G., Mol. Phys. 32 (1976) 1609. 1625.

[3] Champion, J. P., Can. J. Phys. 55 (1977) 1802-1828.

[4] Champion, J. P. et Berger, H., J. Physique 36 (1975) 135-139.

[5] Blatherwick, R. D., Goldman, A., Lutz, B. L., Silvaggio, P. M. et Boese, R. W., Appl. Opt. 18 (1979) 3798-3804.

[6] Pierre, C., Pierre, G., Champion, J. P., Fontanella, J. C. et Delplanque, M., J. Physique 41 (1980) 393-402.

[7] Lutz, B. L., Silvaggio, P. M. et Boese, R. W., Astrophys. J. 227 (1979) 334-337.

[8] Amat, G. et Nielsen, H. H., J. Chem. Phys. 36 (1962) 18591865.

[9] Restelli, G. et Cappellani, F., J. Mol. Spectrosc. 78 (1979) 161-169.
[10] Pinson, P. et Dupré-Maquaire, J., J. Mol. Spectrosc. 78 (1979) $170-174$

[11] Champion, J. P. et Pierre, G., J. Mol. Spectrosc. 79 (1980) 255-280.

[12] Tarrago, G., Dang Nhu, M., Poussigue, G., Guelachvili. G. et Аміот, C., J. Mol. Spectrosc. 57 (1975) 246-263.

[13] Pierre, G. et Champion, J. P., J. Physique Lett. 38 (1977) L-311 - L-316.

[14] Gray, D. L., Robiette, A. G. et Johns, J. W. C., Mol. Phys 34 (1977) 1437-1453.

[15] Champion, J. P., Pierre, G., Berger, H. et Cadot, J., J. Mol Spectrosc. 79 (1980) 281-294.

[16] Gray, D. L. et Robiette, A. G., Mol. Phys. 37 (1979) 1901 1920.

[17] Pierre, G. et Champion, J. P., en préparation. 